\title{
Influence of Primary Stenosis on Secondary One and Vice Versa in case of Double Stenoses
}

\author{
D. K. Mandal ${ }^{1}$, N. K. Manna ${ }^{2}$ and S. Chakrabarti ${ }^{3 \dagger}$ \\ ${ }^{1}$ Dept. of Basic Science \& Humanities, College of Engg. \& Management, Kolaghat, P.O: K.T.P.P. Township, \\ Midnapore (E) - 721 171, West Bengal, India, \\ ${ }^{2}$ Dept. of Mech. Engg., Jadavpur University, Kolkata - 700 032, West Bengal, India \\ ${ }^{3}$ Dept. of Mech. Engg., Bengal Engineering and Science University, Shibpur, Howrah - 711 103, West Bengal, India. \\ †Corresponding Author Email: somnathbec@rediffmail.com
}

(Received May 12, 2009; accepted April 2, 2010)

\begin{abstract}
Numerical solutions of the steady viscous flow in the neighborhood of different double stenoses are obtained under laminar flow conditions with the motivation for modeling blood flow through stenosed artery formed due to arterial disease. The flowing blood is considered to be incompressible and Newtonian. A finite volume method has been employed to solve the governing equations. The dynamics of flow features have been studied by wall pressure, streamline contour, and wall shear stress distributions for all models. The results have demonstrated that when the shapes of stenosis change at primary stenosis keeping no change in the shape of secondary stenosis, the impact of changes in primary stenosis on secondary one is noted to be more, whereas, no impact of primary stenosis on secondary stenosis and vice versa is observed in case of changes in the shapes of secondary stenosis keeping no change in the shape of primary stenosis. When Reynolds number changes, the impact of changes in primary stenosis on secondary one is also noted to be higher.
\end{abstract}

Keywords: Wall pressure, Streamline contour, Peak wall shear stress, Reynolds number.

\section{NOMENCLATURE}

\begin{tabular}{|c|c|}
\hline A & Area at any section, $\left[\mathrm{m}^{2}\right]$ \\
\hline $\mathrm{D}$ & Dia of the artery, $[\mathrm{m}]$ \\
\hline $\mathrm{h}_{\mathrm{f}}$ & Depth of the restriction, $[\mathrm{m}]$ \\
\hline $\mathrm{L}_{\mathrm{i}}$ & $\begin{array}{l}\text { Inlet length (i.e., length between inlet } \\
\text { section and restriction- } 1),[\mathrm{m}]\end{array}$ \\
\hline Lex & $\begin{array}{l}\text { Exit length (i.e., length between } \\
\text { restriction-2 and exit section), [m] }\end{array}$ \\
\hline $\mathrm{Lr}$ & Reattachment length, $[\mathrm{m}]$ \\
\hline Ls & $\begin{array}{l}\text { Stricture length or length of } \\
\text { stenosis, }[\mathrm{m}]\end{array}$ \\
\hline $\mathrm{P}$ or $\mathrm{p}$ & Static pressure, $\left[\mathrm{Nm}^{-2}\right]$ \\
\hline $\mathrm{Pw}$ & Wall pressure, [Nm-2] \\
\hline \multirow[t]{2}{*}{ PR } & Percentage of restriction \\
\hline & or Percent stenosis $=\frac{2 h_{f}}{D} \times 100 \%$ \\
\hline $\mathrm{R}$ & Restriction \\
\hline $\operatorname{Re}$ & Reynolds Number \\
\hline $\mathrm{S}$ & Restriction spacing, $[\mathrm{m}]$ \\
\hline
\end{tabular}

\section{INTRODUCTION}

The study of fluid flow through wavy boundaries is of great interest to engineers and researchers because of the importance it plays in phenomena such as: the

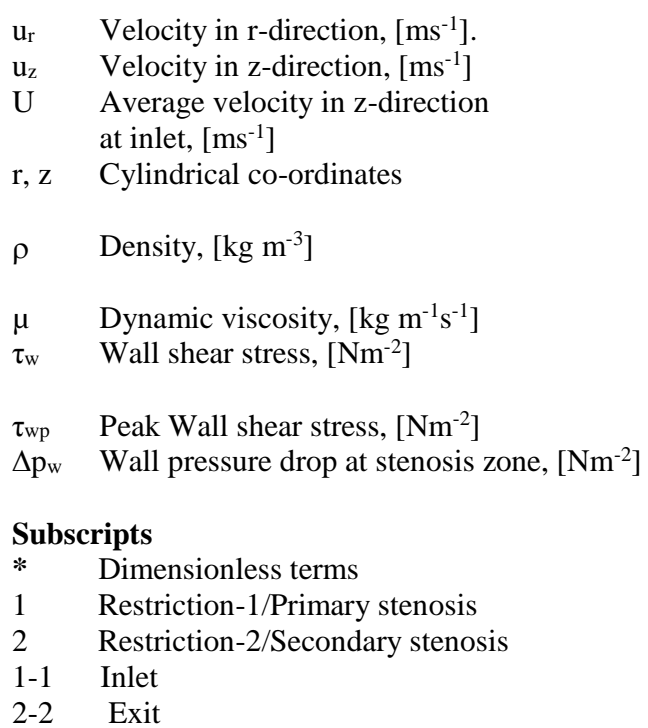

generation of wind waves on water; the stability of a liquid film in contact with a gas stream; fluid flow in pipes with fittings, and blood flow through arteries 
under stenotic conditions. This type of study may also help to the bioengineers who are engaged in the design and construction of improved artificial organs.

Atherosclerosis is a progressive disease characterized by localized plaques that form within the artery wall. It characterizes the hardening and thickening of the arterial wall. With the progress of disease, the passage area of the artery reduces significantly so that the normal flow of blood is severely impaired. The root causes to the formation of atherosclerotic lesions are still unknown but it is well established that the fluid dynamical parameters play an important role in the genesis of the disease. The formation of plaque in artery is closely related with the pressure distribution, the boundary layer separation, and high and low arterial wall stresses in the arterial constriction. It is also recognized that instead of a single stenosis, multiple stenoses may also develop in series in an arterial segment (see Pincombe and Mazumdar 1990; Moore 1997). These constrictions in diseased vascular tube occur because the formation of the primary constriction produces recirculating flow at the downstream of the constriction depending on percent stenosis, stenosis length, restriction spacing, flow Reynolds number etc. This recirculating flow in time helps or assists particle accumulation and formation of a secondary constriction.

Quite a good number of theoretical as well as experimental studies related to blood flow through constricted arteries have been continually persued by the researchers for single as well as multiple restrictions. For multiple restrictions, Solzbach et al. (1987) have investigated experimentally the influence of stenosis geometry, such as percentage lumen area reduction, length, exit angle and eccentric shape on the fluid dynamics of the post stenosis flow considering rectangular shaped restriction. Dreumel and kuiken (1989) have studied numerically and experimentally the flow through trapezoidal shaped double constrictions. Liepsch et al. (1992) have studied experimentally the influence of stenotic geometry under steady flow condition in four cylindrical stenotic models with rectangular cross section. Tandom et al. (1993) have made an attempt to analyze the characteristics of the blood flow through an artery suffering with axisymmetric double stenoses in series. The stenoses are cosine shaped and identical with having each percentage of restriction of $20 \%$. The stricture length $(\mathrm{Ls} *)$ of each restriction is 1.0 and the restriction spacing $\left(\mathrm{S}^{*}\right)$ between two restrictions is 1.25 . The effects of overlapping stenosis on arterial flow problem have been carried out analytically by Chakravarty and Mandal (1994) and Layek et al. (2009). The steady laminar flow fields in the neighborhoods of two consecutive Gaussian shaped restrictions have been studied by Lee (1994) for Reynolds number in the range of 5 to 200, considering the fixed PR of $50 \%$ at the upstream restriction and different PR varying from $20 \%$ to $60 \%$ in the downstream restriction. Damodoran et al. (1996) have studied numerically the steady laminar flow through tubes with four constrictions. A numerical study of an unsteady laminar flow in a double contour shaped constricted 3D vessel has been carried out by Ratish Kumar et al. (2002). In their study, they have considered Reynolds number in the range of 100-1200 and the spacing between the constrictions as 1, 2, 3, 4 and $\infty$ by keeping a fixed PR of $50 \%$ for each constriction, and Strouhal's number of 0.0124. Further, Lee (2002) has studied numerically the effects of steady flow through double similar symmetrical bell-shaped identical constrictions. They have performed the study for percentage of restrictions of $33.3 \%, 50 \%$ and $66.67 \%$, and the Reynolds numbers in the range of 5 to 400 , with a fixed dimensionless restriction spacing of 1.0. Lee (2005) has studied the flow fields in the neighborhood of double constrictions in a circular cylindrical tube for dimensionless spacing of 1, 2, 3 with same PR of $50 \%$ for each restriction. The effects of multiple constrictions on central line velocity, pressure distribution, wall shear stress, and stream lines at different Reynolds numbers are investigated by Layek et al. (2005).

From the brief review of literature, it is evident that most of the researchers have considered the influence of multiple restrictions on the flow keeping fixed stricture length of each restriction. They have also considered that the PR of stenosis increases at same stricture length. During progression of the disease, it seems quite unnatural that the shape of stenosis increases in the $r$ direction by increasing percentage of restriction only. Instead, the shape of stenosis should increase in $r$ and $\mathrm{z}$ directions both. Since the downstream of stenosis is the prone zone for aggravation, the length of stenosis may change more in the downstream than the upstream of stenosis. This phenomenon has been considered in our work. In this work, the shape is considered to become asymmetric one during the progression of the disease. Accordingly, the shape is allowed to change in $\mathrm{r}$ and $\mathrm{z}$ directions both. Moreover, no one has considered multiple stenoses, where first restriction's PR changes and PR of second restriction is fixed.

Therefore, in our present study, we have considered two separate cases. Firstly, the shape of first stenosis changes asymmetrically, the shape of second restriction remains constant. Secondly, the first restriction is fixed, the shape of second restriction changes asymmetrically. Finally, an attempt has been made to study the influence of asymmetric configurations of stenosis on the flow characteristics in each case with comparisons between two cases. The considered flow characteristics are wall pressure, streamline contour and wall shear stress. The effect of Reynolds number on the above flow parameters also has been addressed in this study.

\section{MATHEMATICAL Formulation}

\subsection{Governing Equations}

A schematic diagram of the computational domain is illustrated in Fig.1. It shows the computational domain with Gaussian shaped restrictions. This bell-shaped stenosis geometry is modeled mathematically as $r=0.5-h_{f} \exp \left(-\frac{4 m^{2} z^{2}}{L_{s}{ }^{2}}\right)$, (see Misra and Shit 2006).

Where, $\mathrm{m}$ is a parametric constant. The flow under consideration has been assumed to be steady, twodimensional, laminar and axisymmetric. Since the development of arteriosclerosis in arteries reduce the 


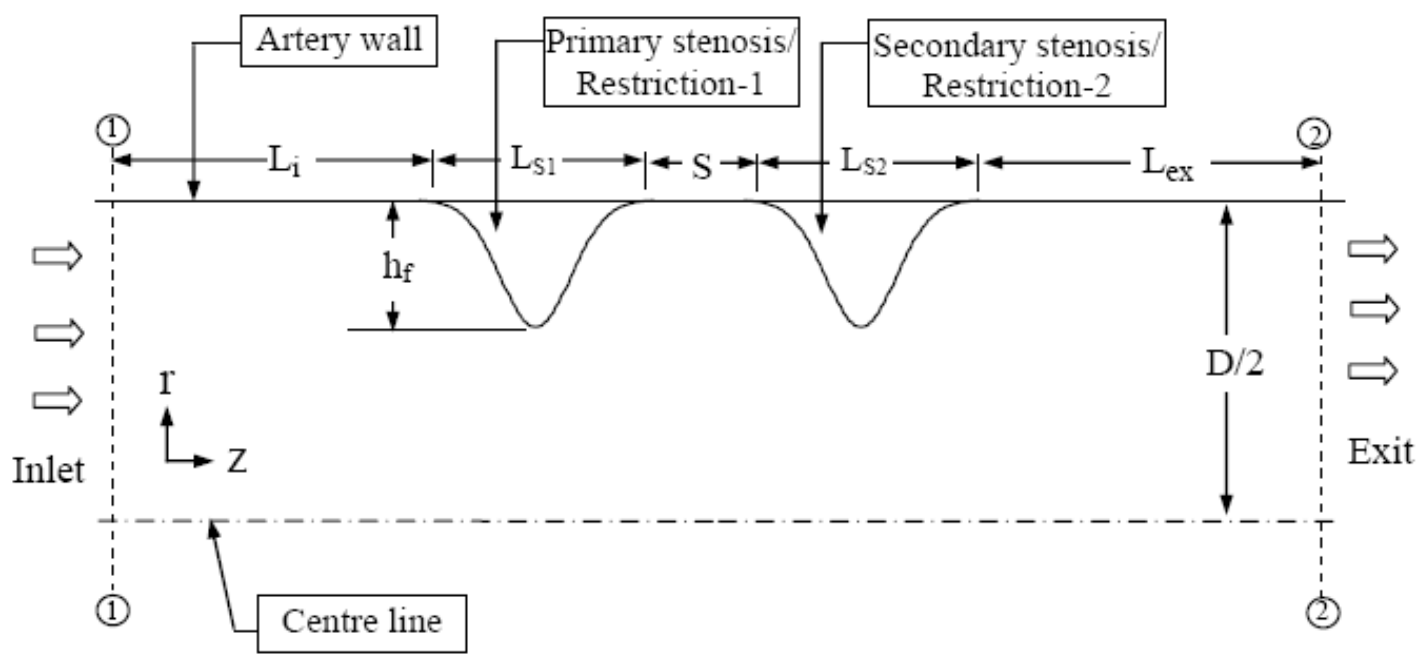

Fig. 1. Computational domain

elastic property of the arterial wall, therefore, in our study, the vessel wall has been considered as rigid tube, (see Liu et al. 2004). It is established that for the diameter of artery $\geq 0.2 \mathrm{~mm}$, shear rate becomes very high which leads for the consideration of blood as a Newtonian fluid for coronary artery, whose diameter is approximately $0.3 \mathrm{~cm}$, (Research Training Network on Mathematical Modeling for Haemodynamics, HaeModel, http://iacs.epfl.ch/cmcs/Haemodel.php3).

Therefore, in our study, the fluid, i.e. blood, is considered to be Newtonian and incompressible. The flow of blood in the blood vessels, like the flow of liquids in narrow rigid tubes, is normally considered laminar, (see Ganong 2001). From the literatures of Andersson et al. (2000) and Back et al. (2000), it is established that physiological flow range of Reynolds number is 100 to 400 for main coronary artery of human being in the laminar zone. Bertolotti et al. (2001) have also mentioned that the critical Reynolds number of the flow of blood through coronary artery is in between 300 to 400 , above which the flow becomes transitional and turbulent at $75 \%$ stenosed condition. Therefore, the consideration of flow as laminar at our considered Reynolds number upto 400 seems to be reasonable.

The following dimensionless variables are defined to obtain the governing conservation equations in the nondimensional form;

Lengths: $\quad r^{*}=r / D, \quad z^{*}=z / D, \quad L_{i}^{*}=L_{i} / D$,

$L_{e x}^{*}=L_{e x} / D, \quad L_{S 1}^{*}=L_{s 1} / D, \quad L_{S 2}^{*}=L_{s 2} / D$,

$h_{f}^{*}=h_{f} / D, S^{*}=S / D$.

Velocities: $u_{r}{ }^{*}=u_{r} / U, u_{z}{ }^{*}=u_{z} / U$.

Pressure: $p^{*}=p / \rho U^{2}$.

With the help of these variables, the mass and momentum conservation equations are written as follows,

$\frac{\partial}{r^{*} \partial r^{*}}\left(r^{*} u_{r}^{*}\right)+\frac{\partial}{\partial z^{*}}\left(u_{z}^{*}\right)=0$

$$
\begin{aligned}
& \frac{\partial\left(r^{*} u_{r}^{*} u_{r}^{*}\right)}{r^{*} \partial r^{*}}+\frac{\partial\left(u_{z}{ }^{*} u_{r}^{*}\right)}{\partial z^{*}}=-\frac{\partial p^{*}}{\partial r^{*}}+\frac{1}{\operatorname{Re}}\left(\frac{\partial}{\partial r^{*}}\left(\frac{\partial\left(r^{*} u_{r}\right)}{r^{*} \partial r^{*}}\right)+\frac{\partial^{2} u_{r}{ }^{*}}{\partial z^{* 2}}\right) \\
& \frac{\partial\left(r^{*} u_{r}{ }^{*} u_{z}{ }^{*}\right)}{r^{*} \partial r^{*}}+\frac{\partial\left(u_{z}{ }^{*} u_{z}{ }^{*}\right)}{\partial z^{*}}=-\frac{\partial p^{*}}{\partial z^{*}} \\
& +\frac{1}{\operatorname{Re}}\left(\frac{\partial}{r^{*} \partial r^{*}}\left(r^{*} \frac{\partial u_{z}{ }^{*}}{\partial r^{*}}\right)+\frac{\partial^{2} u_{z}^{*}}{\partial z^{*}}\right)
\end{aligned}
$$

Where, the flow Reynolds number, $\operatorname{Re}=\rho U D / \mu$.

\subsection{Boundary Conditions}

Four different types of boundary conditions have been applied to the present problem. They are as follows,

1. At the walls: No slip condition, i.e. $u_{r}{ }^{*}=0, u_{z}{ }^{*}=0$.

2. At the inlet: Axial velocity has been specified and the transverse velocity has been set to zero, i.e. $u_{z}{ }^{*}=$ specified,$\quad u_{r}{ }^{*}=0$. Fully developed flow condition has been specified at the inlet, i.e., $u_{z}^{*}=2.0\left[1-\left(2 r^{*}\right)^{2}\right]$.

3. At the exit: Fully developed condition has been assumed and hence gradients have been set to zero, i.e., $\partial u_{z}^{*} / \partial x^{*}=0, u_{r}^{*}=0$.

4. At the line of symmetry: The normal gradient of the axial velocity and the transverse velocity have been set to zero, i.e., $\partial u_{z}{ }^{*} / \partial r^{*}=0, u_{r}{ }^{*}=0$.

\subsection{Numerical Procedure}

The governing Eqs. (1), (2) and (3) have been solved numerically by an in-house CFD code developed using integral approach of the finite volume method on a nonuniform staggered grid following SIMPLER algorithm (see Pathankar 1980). The third-order upwind scheme has been used for advective part. The discretized equations have been solved using Tri-diagonal Matrix Algorithm (TDMA) with Alternate Direction Implicit (ADI) scheme. The convergence of the iterative scheme has been achieved when the normalized residuals for 
mass and momentum equations summed over the entire calculation domain will fall below $10^{-7}$.

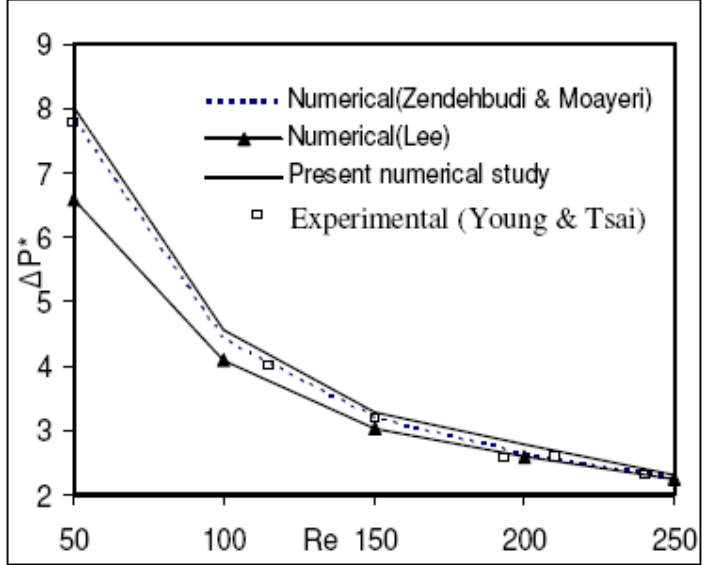

Fig. 2. Comparisons with available results of others for single restriction with PR of $33.33 \%$
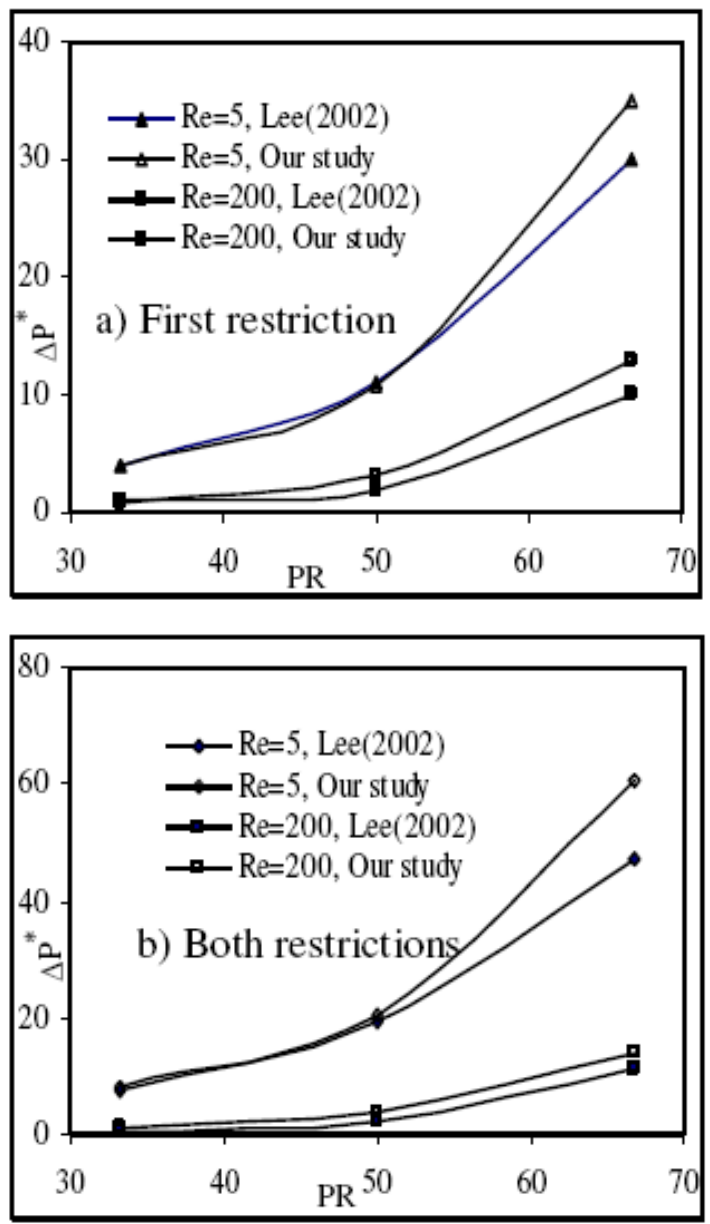

Fig. 3. Comparisons with the result of wall pressure drops across restrictions for $\mathrm{Re}=5$ and 200 for $\mathrm{PR}=33.33 \%, 50 \%$ and $66.67 \%$

In the computation, flow has been considered fully developed at inlet and exit, and therefore, the nondimensional total length of computational domain has been chosen to be 200 and the inlet length has been chosen to be 50. After grid independence test, finally the numerical mesh comprised of $449 \times 65$ grid nodes in $\mathrm{z}$ and $\mathrm{r}$ direction has been considered in the present work.

\subsection{Validation of Computational Results}

For the purpose of validations of our results, initially the computation has been carried out for a single restriction considering cosine shaped geometry of $56 \%$ area reduction for different values of Reynolds number, as was done by Young and Tsai (1973) during their experimental work. This study has been carried out for the stricture length of 4.0 , percentage of restriction of $33.33 \%$ and the non-dimensional length of the computational domain of 16 . The results of dimensionless pressure drop, obtained from our computation, have been compared with the experimental work of Young and Tsai (1973), and the numerical results of Zendehbudi and Moayeri (1999), and Lee (2002) in Fig. 2. Comparisons have showed a very good agreement. For the purpose of validations of our results in the case of flow through double bell shaped restrictions, we have carried out the computations of wall pressure drops across the restrictions at $\mathrm{Re}$ of 5.0 and 200 to compare our results with the results of Lee (2002). During the computation, the magnitude of $\mathrm{Ls}^{*}$ and $\mathrm{S}^{*} \quad\left(\mathrm{Ls}^{*}{ }_{1}=1.0, \mathrm{~S}^{*}=0.0\right.$, $\mathrm{Ls}^{*}{ }_{2}=1.0$ ) have been chosen same as was considered by Lee (2002). The variations of pressure drop across restriction-1 and both restrictions have been shown in Figs. 3a and $3 \mathrm{~b}$ respectively. In general, pressure drop across restrictions shows a good agreement except the small deviation at higher percentage of restriction.

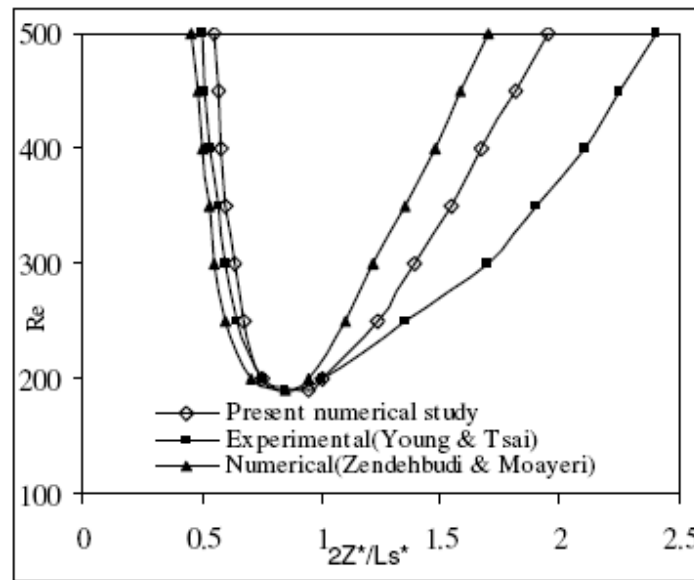

Fig. 4. Separation-reattachment curves for single restriction

Further, the separation-reattachment curves for the same single restriction (Young \& Tsai 1973) from different studies are shown in Fig. 4, where z-axis is represented as was considered by Young \& Tsai (1973). There is a good agreement between present result and numerical results of Zendehbudi \& Moayeri (1999). Apparently, the large discrepancy with experimental results of Young \& Tsai (1973) has been observed, as the reattachment lengths are difficult to measure experimentally. To check the validity of our numerical results in case of double restrictions, the separationreattachment curves for the first restriction as well as second restriction are compared with Lee (1994) for $\mathrm{Ls}^{*}{ }_{1}=1.0, \mathrm{PR}_{1}=50 \%, \mathrm{~S}^{*}=0.0, \mathrm{PR}_{2}=60 \%$, and $\mathrm{Ls}_{2}=1.0$, 


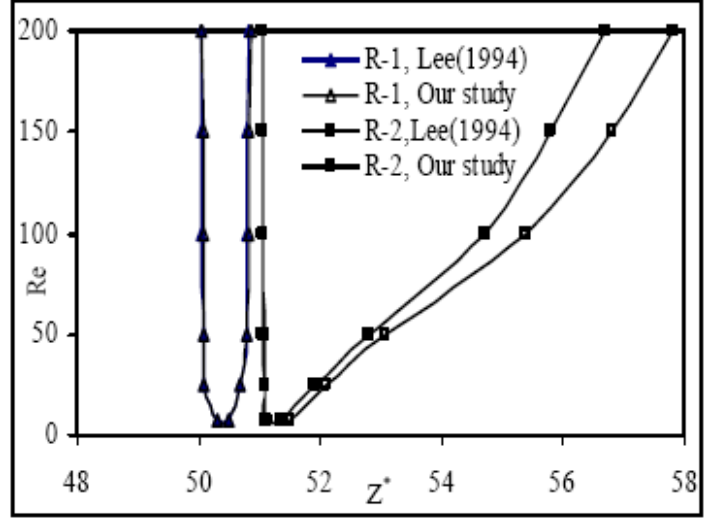

Fig. 5. Separation-reattachment curves for double restrictions

shown in Fig. 5. This shows a good agreement except the small deviation at higher Reynolds number in case of second restriction.

Table 1 Details of different shapes.

\begin{tabular}{|c|c|c|c|c|c|}
\hline Model & $\mathrm{Ls}^{*}{ }_{1}$ & $\mathrm{PR}_{1}$ & $\mathrm{Ls}^{*}{ }_{2}$ & $\mathrm{PR}_{2}$ & Case \\
\hline M1 & 0.2 & $30 \%$ & \multirow{4}{*}{0.2} & \multirow{4}{*}{$30 \%$} & \multirow{4}{*}{ Case 1} \\
\hline AS1 & 0.3 & $50 \%$ & & & \\
\hline $\mathrm{AS} 2$ & 0.4 & $70 \%$ & & & \\
\hline AS3 & 0.5 & $90 \%$ & & & \\
\hline M1 & \multirow{4}{*}{0.2} & \multirow{4}{*}{$30 \%$} & 0.2 & $30 \%$ & \multirow{4}{*}{ Case 2} \\
\hline AS4 & & & 0.3 & $50 \%$ & \\
\hline AS5 & & & 0.4 & $70 \%$ & \\
\hline AS6 & & & 0.5 & $90 \%$ & \\
\hline
\end{tabular}

\subsection{Problem Formulation}

The formulations of different shapes are shown in Fig. 6 and the details of different shapes are presented in Table 1. Initially we have considered a double stenoses like the model: M1. Both restrictions of this model are similar symmetrical (about the centre of restriction) having same stricture length of 0.2 and $30 \%$ restriction each. In case-1, the aggravation takes place in the first stenosis mainly. Then the shape of first restriction of multi-stenoses changes from M1 to AS1, AS1 to AS2, and AS2 to AS3. Here the shape of second restriction remains same like the model: M1. In case-2, the shape of first restriction remains same like the model: M1. The aggravation takes place in the second stenosis, the shape changes from M1 to AS4, AS4 to AS5 and AS5 to AS6 accordingly.

\section{RESUlts AND Discussion}

Numerical simulations have been carried out to model the steady flow of blood through a coronary artery with double bell shaped stenoses. During numerical investigation; firstly, the effect of different shaped double stenoses (for case-1 and case-2) on flow characteristics, e.g. wall pressure drop, streamline contour and peak wall shear stress; secondly, the effect of Reynolds number on the above flow parameters have been carried out for two typical shaped stenoses (Model: AS1 and AS4).

\subsection{Effect of Shape of Stenoses}

In this subsection, the effect of different shaped double stenoses like case- 1 and case- 2 on wall pressure, streamline contour and wall shear stress has been investigated and comparisons between the two cases have been outlined. The non-dimensional restriction spacing between two restrictions has been considered to be 4.0 for initial condition of Model: M1. So, in case-1, the $S^{*}$ changes accordingly as $3.9,3.8$ and 3.7 for the models AS1, AS2, and AS3 respectively. In case-2, the $\mathrm{S}^{*}$ remains same as 4.0.
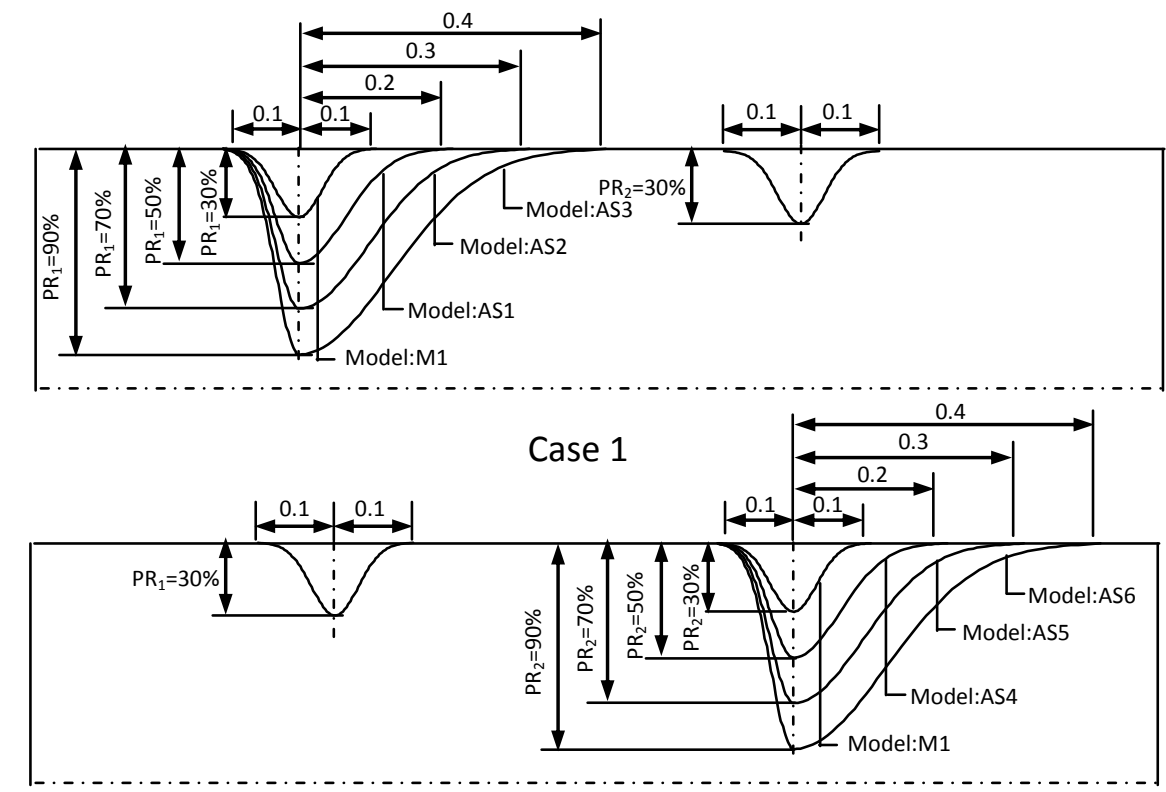

Case 2

Fig. 6. Different shapes 
D. K. Mandal et al. / JAFM, Vol. 4, No. 4, pp. 31-42, 2011.
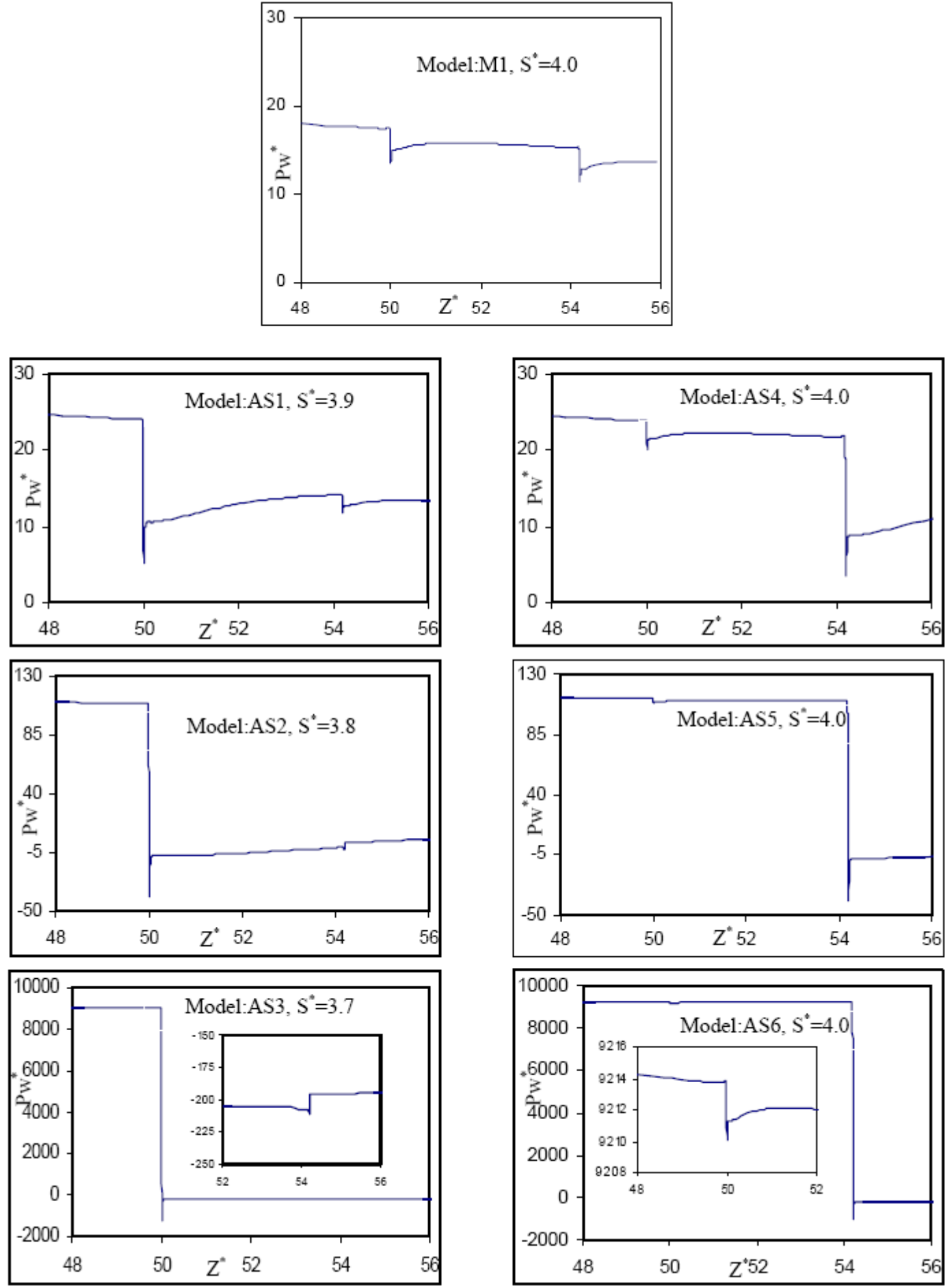

Fig. 7a. Variations of wall pressure for $\mathrm{Re}=100$ for case- 1 and case-2.

The parameters during the study are identified as,

(i) Reynolds number $=100$.

(ii) Percentage of restriction $=30 \%, 50 \%, 70 \%$ and $90 \%$.

(iii) Stricture length $=0.2,0.3,0.4,0.5$.

\subsubsection{Variations of Wall Pressure}

The variations of wall pressure have been shown in Fig. 7a for Reynolds number of 100 for case- 1 and case-2. The inset figures in model: AS3 and model: AS6 show the enlarged view of variation of wall pressure at second restriction for model AS3 and first restriction of model: AS6 respectively. Here, nondimensional pressure drop at first stenosis $\left(\Delta \mathrm{Pw}^{*}{ }_{1}\right)$ increases with the change of considered models from $\mathrm{M} 1$ to AS3 in case-1. In this type of aggravation, $\Delta \mathrm{Pw}^{*}{ }_{1}$ increases due the high impact of restriction PR compared to the effect of stricture length and restriction spacing. 
D. K. Mandal et al. / JAFM, Vol. 4, No. 4, pp. 31-42, 2011.
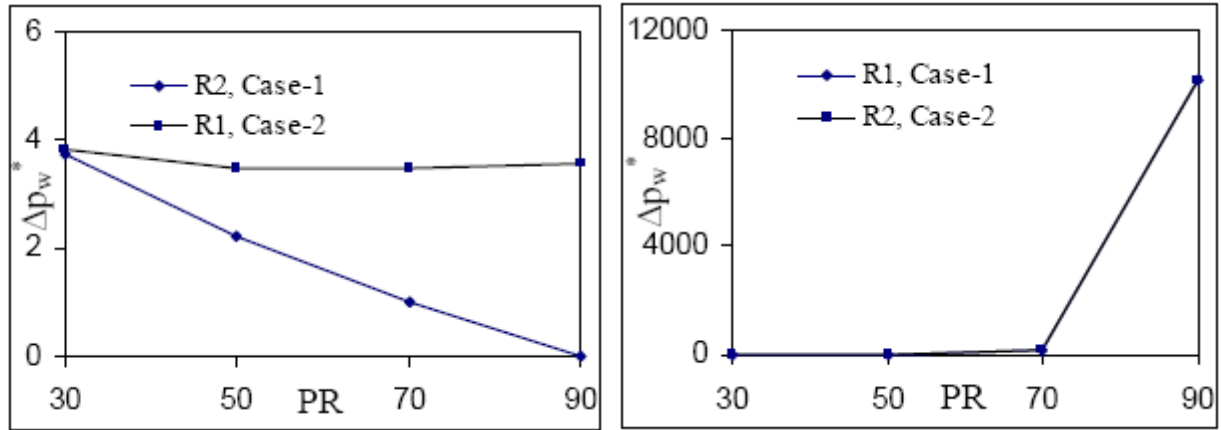

Fig. 7b. Variations of wall pressure drop at stenosis zone for $\mathrm{Re}=100$ for case- 1 and case- 2 .

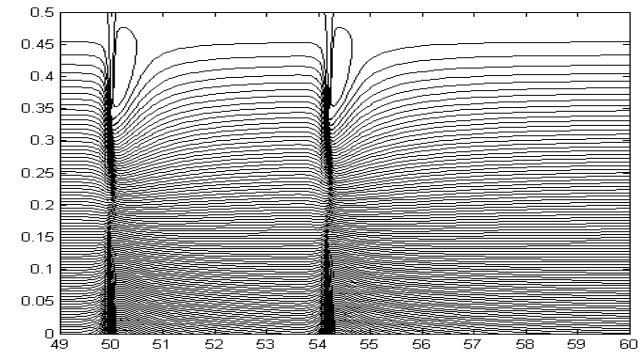

Model:M1, $\mathrm{S}^{*}=4.0$

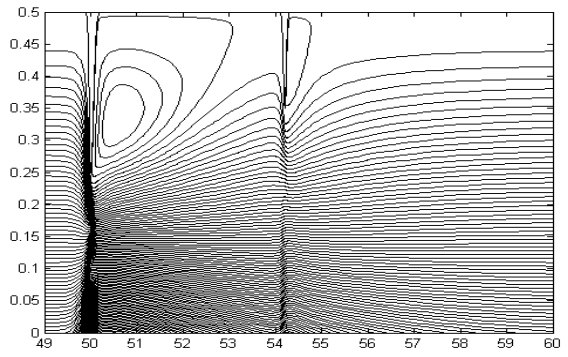

Model:AS1, $\mathrm{S}^{*}=3.9$

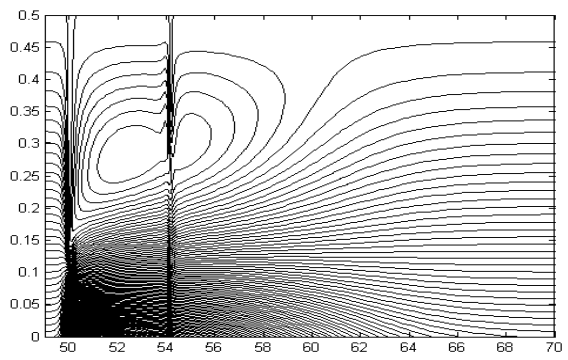

Model:AS2, $\mathrm{S}^{*}=3.8$

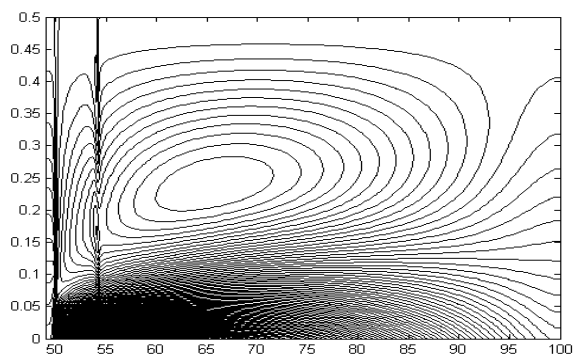

Model:AS3, $\mathrm{S}^{*}=3.7$

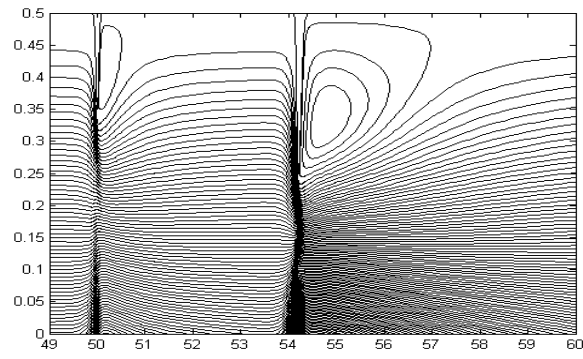

Model:AS4, $\mathrm{S}^{*}=4.0$

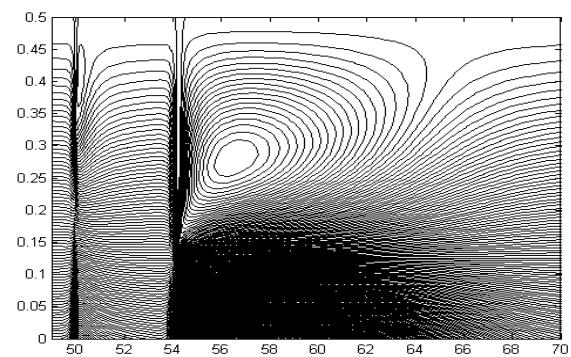

Model:AS5, $\mathrm{S}^{*}=4.0$

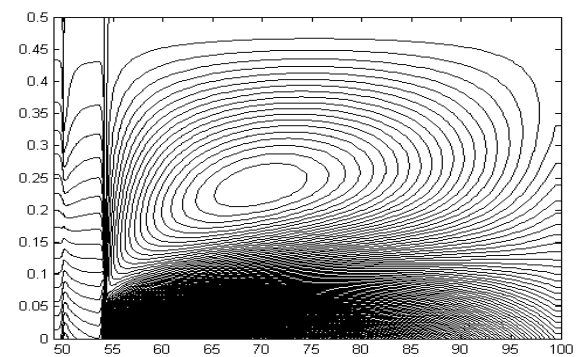

Model:AS6, $\mathrm{S}^{*}=4.0$

Fig. 8. Contours plotting for $\mathrm{Re}=100$ for case- 1 and case- 2 . 
For this case- 1 , it is interesting to note that the $\Delta \mathrm{Pw}^{*} 2$ decreases instead of same shape of second restriction. These happenings may be due to the higher impact of higher length of recirculation bubble at the downstream of first restriction due to higher PR of first restriction. For case-2, no appreciable change in magnitude of $\Delta \mathrm{Pw}^{*}{ }_{1}$ has been noticed when model changes from $\mathrm{M} 1$ to AS6. Pressure drop at second restriction, $\Delta \mathrm{Pw}_{2}{ }_{2}$ increases due to increase in PR of second restriction in this case-2. It is also noted from Fig. 7a that the magnitude of pressure drop at first stenosis in case-1 and pressure drop in second restriction in case- 2 are more or less same. All these observations have been substantiated with the help of Fig.7b.

Since, there is chance of tearing action on arterial wall due to low wall pressure, which causes of damaging the endothelium and adjacent wall layers, with subsequent thickening of the intima and eventual plaque development, therefore, the impact due to wall pressure drop on the chance of progression of disease increases at first restriction in case- 1 and second restriction in case-2, and this quantum of magnitude of impact remains same for both the cases. This impact decreases for second restriction in case-1, and remains same for first restriction in case-2.

\subsubsection{Variations of Streamlines Contour}

The variations of streamline contour have been shown in Fig. 8 for Reynolds number of 100 in case- 1 and case- 2 . In case-1, i, e., when the model changes from M1 to AS3, $\mathrm{Lr}^{*}{ }_{1}$ increases in spite of opposite impact of stricture length on the length of recirculation zone. This indicates higher impact of PR on recirculation length compared to stricture length. In case of models of AS2 and AS3, the inner zone between two restrictions is fully filled up by recirculation bubble. Moreover, it is noted that the recirculating eddy between the two constrictions spreads beyond the second restriction and merges with the eddy that formed behind it. This supplements our earlier prediction stated in the wall pressure drop analysis. For case-2, the recirculation zone formed behind the first restriction has been observed to be more or less same. This zone increases for second restriction as PR increases.

The recirculation zone is the cause of stagnation of blood stream and it allows platelets and fibrin to form a mesh at the inner wall in this zone by trapping lipid particles to form atheromatous plaque. So, the plaque deposition zone at $\mathrm{R}_{1}$ increases for case- 1 . This zone is noted to be increasing with increase in $\mathrm{PR}_{1}$ considering both impact of $R_{1}$ and $R_{2}$ for the models of AS2 and AS3. In case- 2 , the plaque deposition zone at $R_{1}$ does not change appreciably. This zone has been noted to be increasing at $\mathrm{R}_{2}$.

\subsubsection{Variations of Wall Shear Stress}

The non-dimensional wall shear stress at any position is computed with the help of the following expression:

$\tau_{w}^{*}=\frac{\tau_{w}}{\tau_{w \text { ref }}}$ where, $\tau_{\mathrm{w}}$ is wall shear stress and $\tau_{\text {wref }}$ is the reference wall shear stress, which is considered as the wall shear stress far away from the stenosis.
Figure 9a shows the variations of wall shear stress for Reynolds number of 100 for case- 1 and case-2. Inset figures in case of model: AS3 and AS6, show the variation of wall shear stress at the second restriction $\left(\mathrm{R}_{2}\right)$ in case-1 and first restriction in case-2 respectively. It is evident from the figure that the magnitude of $\tau_{\mathrm{wp}}{ }^{*} 1$ increases as $\mathrm{PR}_{1}$ increases in case- 1 . The magnitude of $\tau_{\mathrm{wp}}{ }^{*} 2$ decreases in case- 1 and finally the effect of negative wall shear stress appears, although there is no change in the shape of second restriction. This happens due to merging of recirculation eddy of the first restriction with the eddy formed behind the second restriction. For case-2, the developed peak wall shear stress does not change appreciably at $\mathrm{R}_{1}$ and this stress increases at $\mathrm{R}_{2}$ as $\mathrm{PR}_{2}$ increases. It is also noted that the magnitude of peak wall shear stress at first stenosis in case- 1 and peak wall shear stress in second restriction in case- 2 are more or less same. All the above mentioned observations have been supplemented by Fig. 9 b.

It is known that high wall shear stress damages the vessel wall and causes intimal thickening and low wall shear stress causes the mass transportation across the arterial wall. Therefore, the damaging chance of arterial wall due to peak wall shear stress at $\mathrm{R}_{1}$ increases in case-1. This impact of peak wall shear stress on the phenomenon for second restriction decreases with the increase in percentage of restriction of first restriction. Finally at higher $\mathrm{PR}_{1}$, the impact of low wall shear stress appears and chance of aggravation of the disease takes place accordingly. For case-2, this impact at first restriction does not change appreciably and the said impact increases at second restriction. It can also be said that the impact due to peak wall shear stress remains same at first restriction in case- 1 and second restriction in case-2 both.

\subsection{Effect of Reynolds Number}

In this subsection, the simulation of flow characteristics for the models AS1 with $S^{*}=3.9$, and AS4 with $S^{*}=4.0$ has been carried out for the Reynolds numbers of 100 , 200, 300 and 400.

The variations of wall pressure drop at the restrictions zone, streamline contour, and variations of peak wall shear stress for all Reynolds numbers for considered models of AS1 and AS4 have been shown in Figs. 10a, $10 \mathrm{~b}$ and 10c. From the figure of wall pressure drop, it is noted that the magnitude of non-dimensional pressure drop decreases with the increase in Reynolds number for all considered models and for both restrictions (R1 and R2). The magnitude of $\Delta \mathrm{Pw}^{*}{ }_{2}$ for the model AS1 decreases with Re, but after some value of Re this value becomes zero, it may be due to merging of recirculation eddy of first restriction with the recirculating eddy of the second restriction. This is substantiated by the figure of streamline contours (Fig. 10b). From the figure of streamline contour, it is observed that the size of recirculation bubble for both the restrictions increases with Reynolds number for all models. From Fig. 10c, it can be mentioned that the magnitude of peak wall shear stress for first restriction increases with Reynolds number, but this value decreases with Reynolds number for second restriction and attains 
D. K. Mandal et al. / JAFM, Vol. 4, No. 4, pp. 31-42, 2011.
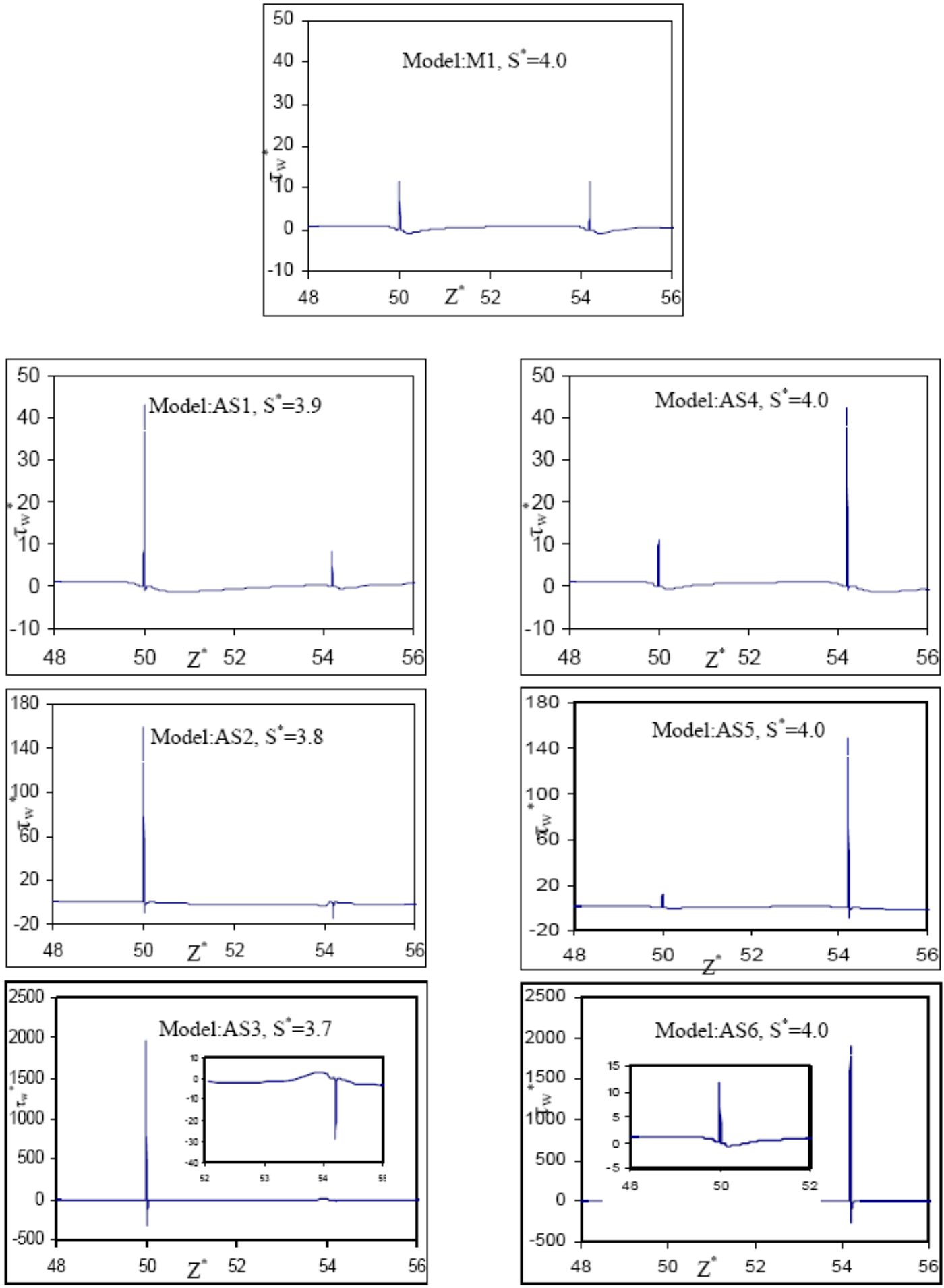

Fig. 9a. Variations of wall shear stress for $\mathrm{Re}=100$ for case- 1 and case- 2 .

negative peak wall shear stress value due to merging of recirculation bubble in case of model: AS1. In case of model: AS4, the magnitude of peak wall shear stress increases with Reynolds number for both the restrictions. The tearing chance of arterial wall due to wall pressure and plaque deposition zone due to recirculation eddy increase with the increase in Reynolds number for both restrictions $\left(\mathrm{R}_{1}\right.$ and $\left.\mathrm{R}_{2}\right)$ for model AS1 and AS4. The chance of damaging of arterial wall due to peak wall shear stress increases with Reynolds number at $\mathrm{R}_{1}$ for AS1 and AS4.

In case of AS1 at $\mathrm{R}_{2}$, the aggravation of disease initially decreases with Re due to peak wall shear stress, but gradually the chance of aggravation of the disease due to low wall shear stress appears finally. In case of. $R_{2}$, the chance of aggravation of the disease due to peak wall shear stress for AS4 increases with Re. 

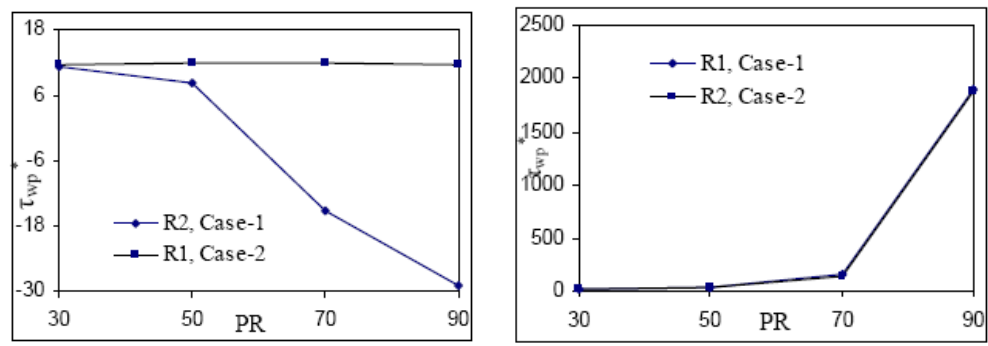

Fig. 9b. Variations of peak wall shear stress for $\mathrm{Re}=100$ for case- 1 and case- 2

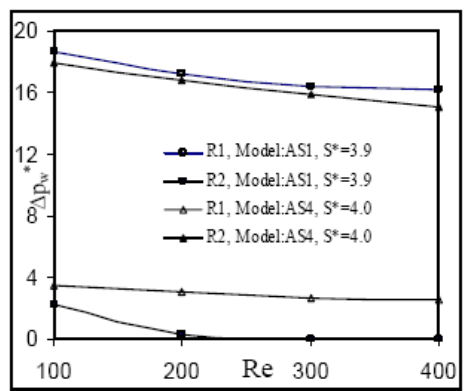

Fig. 10a. Variations of wall pressure drop

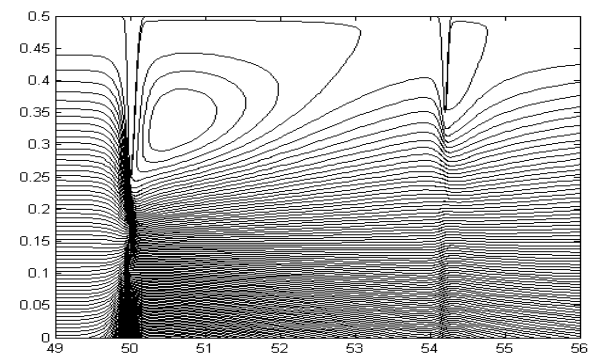

Model: AS1, $\mathrm{S}^{*}=3.9, \mathrm{Re}=100$

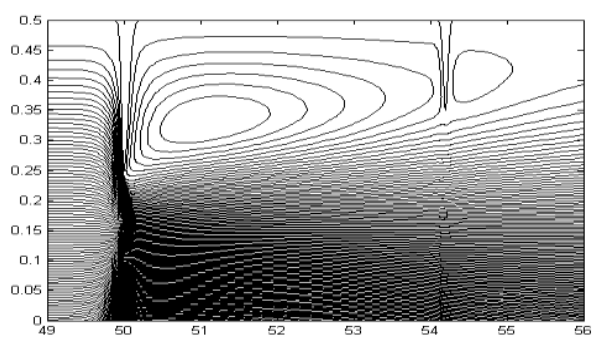

Model: AS1, $\mathrm{S}^{*}=3.9, \mathrm{Re}=200$

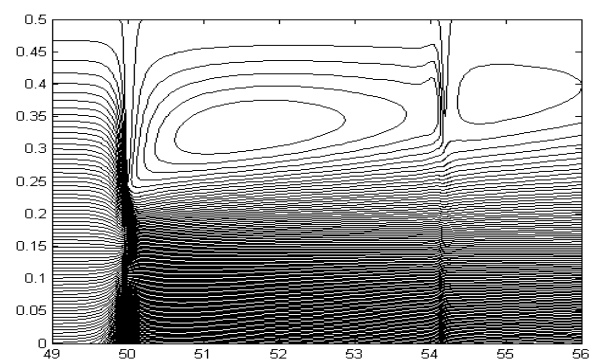

Model: $\mathrm{AS} 1, \mathrm{~S}^{*}=3.9, \mathrm{Re}=300$

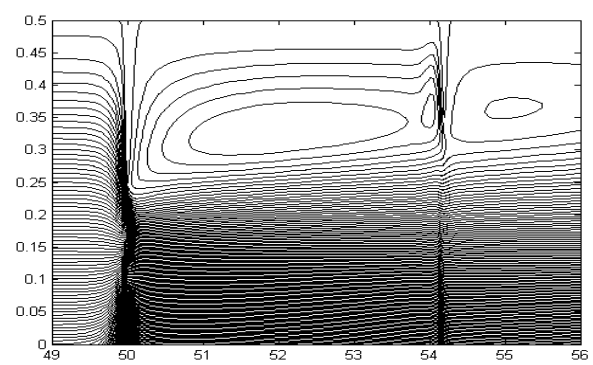

Model: $\mathrm{AS} 1, \mathrm{~S}^{*}=3.9, \mathrm{Re}=400$

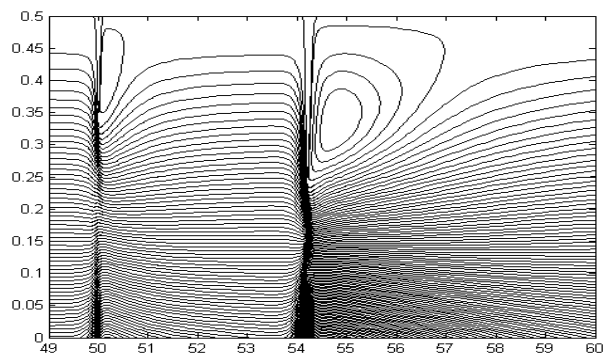

Model: $\mathrm{AS} 4, \mathrm{~S}^{*}=4.0, \mathrm{Re}=100$

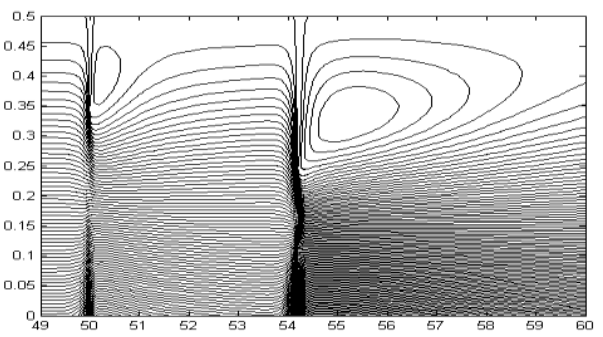

Model: $A S 4, S^{*}=4.0, \operatorname{Re}=100$

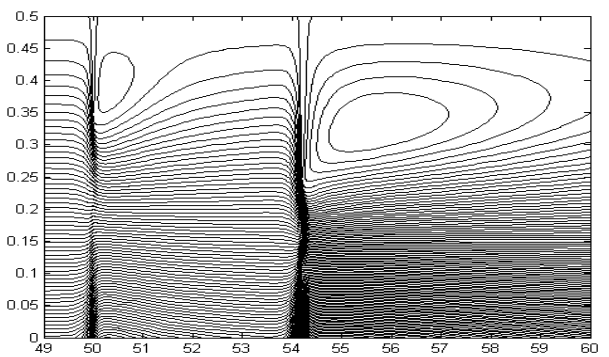

Model: AS4, $\mathrm{S}^{*}=4.0, \mathrm{Re}=100$

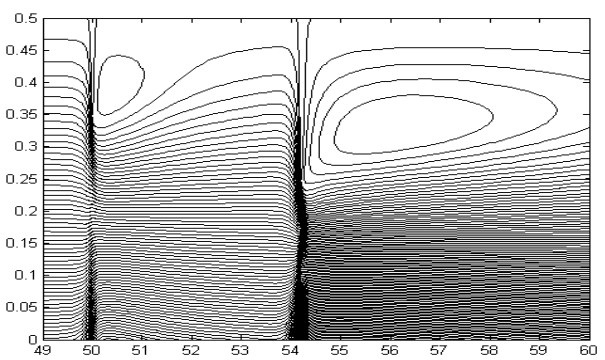

Model: AS4, $\mathrm{S}^{*}=4.0, \mathrm{Re}=100$

Fig. 10b. Contours plotting to show the effect of Re. 


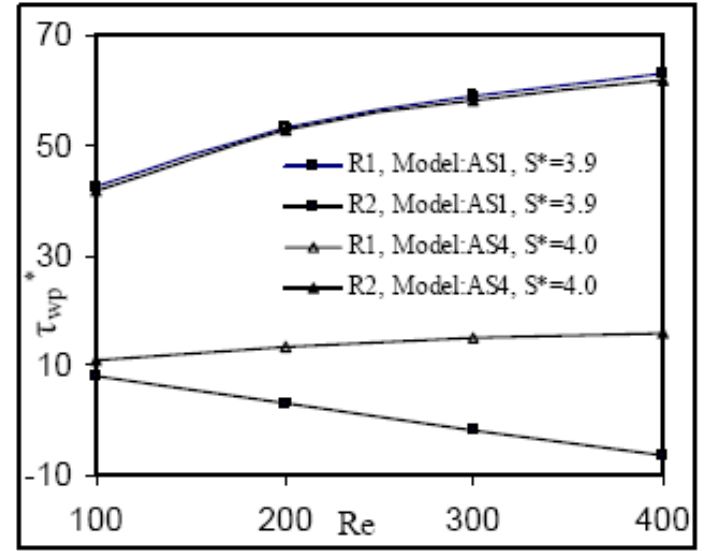

Fig. 10c. Variations of peak wall shear stress.

\section{Conclusions}

In the present numerical study, the effect of different shaped stenoses, and Reynolds number on the flow characteristics like wall pressure, streamline contour and wall shear stress of blood flow through different double bell shaped stenosed coronary artery has been investigated and role of all the flow characteristics on the progression of the disease, atherosclerosis is discussed.

During our study on the effect of different shaped stenoses on flow characteristics and finally on the progression of the disease, it is revealed that the chance of tearing possibilities of arterial wall due to pressure drop, the zone of plaque deposition on the artery wall due to the length of recirculating bubble, and the chance of damaging of arterial wall due to peak wall shear stress increase at first restriction in case- 1 and at second restriction in case-2. The quantum of magnitude of impact also remains same for both the cases. In case-1, the impact due to pressure drop and peak wall shear stress decreases at second restriction. The magnitude of peak wall shear stress becomes negative value at higher percentage of restriction of first restriction in case- 1 . The impact due to pressure drop, reattachment length and peak wall shear stress remains same at first restriction in case-2.

The impact due to pressure drop and recirculating eddy on the progression of the disease increases with Reynolds number for both the restrictions for both the considered models i, e., AS1 and AS4. The impact of peak wall shear stress is also noted to be increasing with Reynolds number for first restriction for both the models. In case of second restriction, this impact due to peak wall shear stress increases for model:AS4. For model:AS1, the impact at second restriction decreases with the final appearance of the effect of low wall shear stress.

Therefore, it can be stated that when the shapes of stenosis change at primary stenosis keeping no change in the shape of secondary stenosis, the impact of changes in primary stenosis on secondary one is noted to be more, whereas, no impact of primary stenosis on secondary stenosis and vice versa is observed in case of changes in the shapes of secondary stenosis keeping no change in the shape of primary stenosis. When Reynolds number changes, the impact of changes in primary stenosis on secondary one is also noted to be higher.

\section{REFERENCES}

Andersson, H.I., R. Halden and T. Glomsaker (2000). Effects of Surface Irregularities on Flow Resistance in Differently Shaped Arterial Stenoses. Journal of Biomechanics 33, 1257-1262.

Back, L.H. and R. K. Banerjee (2000). Estimated Flow Resistance Increase in a Spiral Human Coronary Artery Segment. Journal of Biomechanical Engineering 122, 675-677.

Bertolotti, C., V. Deplano, J. Fuseri and P. Dupouy (2001). Numerical and Experimental models of Post-Operative Realistic flows in Stenosed Coronary Bypasses. Journal of Biomechanics 34, 1049-1064.

Chakravarty, S. and P.K. Mandal (1994). Mathematical Modelling of Blood Flow Through an Overlapping Arterial Stenosis. Mathl. Comput. Modelling 19(1), 59-70.

Damodaran, V., G.W. Rankin and C. Zhang (1996). Numerical Study of Steady Laminar flow through Tubes with Multiple Constrictions using Curvilinear Coordinates. International Journal of Numerical Method in Fluids 23, 1021-1041.

Dreumel, S.C. and G.D.C. Kuiken (1989). Steady Flow through a Double Converging-Diverging Tube Model for Mild Coronary Stenosis. Journal of Biomechanical Engineering 111, 212-221.

Ganong, W.F. (2001). Review of Medical Physiology. Appleton.

Layek, G.C., S. Mukhopadhyay and Sk.A. Samad (2005). Oscillatory flow in a Tube with Multiple Constrictions. Int. J. Fluid Mech. Res. 32, 402419.

Layek, G.C., S. Mukhopadhyay and Rama Subba Reddy Gorla (2009). Unsteady Viscous Flow with Variable Viscosity in a Vascular Tube with an Overlapping Constriction. International Journal of Engineering Science 47, 649-659.

Lee, T.S. (1994). Steady laminar Fluid flow through Variable Constrictions in vascular tube. Journal of fluid Engineering 116, 258-289.

Lee, T.S. (2002). Numerical Study of Fluid Flow through Double Bell-Shaped Constrictions in a Tube. International Journal of Numerical Methods for Heat and Fluid Flow 12(3), 258-289.

Lee, T.S. (2005). Numerical studies of fluid flow through tubes with double constrictions. International Journal of Numerical Methods in Fluids 11 (8), 1113-1126. 
D. K. Mandal et al. / JAFM, Vol. 4, No. 4, pp. 31-42, 2011.

Liepsch, D., M. Singh and M. Lee (1992). Experimental Analysis of the Influence of Stenotic Geometry on Steady flow. Biorheology 29(4), 419-31.

Liu, Guo-Tao., Xian-Ju. Wang, Bao-Quan. Ai and Liang-Gang. Liu (2004). Numerical study of pulsating flow through a tapered artery with stenosis. Chinese Journal of Physics 42(4-1), 401409.

Misra, J.C. and G.C. Shit (2006). Blood Flow Through arteries in a Pathological State: A Theoretical study. International Journal of Engineering Science 44, 662-671.

Moore, K.L. (1990). Clinically Oriented Anatomy. Williiams and Wilkins, Baltimore, MD.

Patankar, S.V. (1980). Numerical Heat Transfer. North Holland.

Pincombe, B. and J. Mazumdar (1997). The effects of post-stenotic dilatations on the flow of a blood analogue through stenosed coronary arteries. Mathl. Comput. Modelling 25(6), 57-70.

Rathish Kumar, B.V., T. Yamaguchi, H. Liu and R. Himeno (2002). A Numerical Study of an Unsteady Laminar Flow in a Doubly Constricted 3D Vessel. Int. J. Numer. Meth. Fluids 38, 11591176.

Solzbach, U., H. Wollschlager, A. Zeiher and H. Just (1987). Effect of Stenotic Geometry on Flow Behaviour Across Stenotic Models. Medical and Biological Engineering and Computing 25, 543550 .

Tandom, P.N., U.V.S. Rana, M. Kawahara and V.K. Katiyar (1993). A model for blood flow through a stenotic tube. Int. J. Biomed. Comput. 32 ,61-78.

Young, D.F. and F.Y. Tsai (1973a). Flow Characteristics in Models of Arterial Stenoses-I. Steady flow. Journal of Biomechanics 6(4), 395410.

Zendehbudi, G.R. and M.S. Moayeri (1999). Comparison of Physiological and Simple Pulsatile Flows Through Stenosed Arteries. Journal of Biomechanics 32, 959-969. 